University of Texas at El Paso

ScholarWorks@UTEP

\title{
9-2014
}

\section{Fuzzy Intervals As Foundation Of Metrological Support For Computations With Inaccurate Data}

\author{
Konstantin K. Semenov \\ Saint-Petersburg State Polytechnical University, semenov.k.k@gmail.com \\ Gennady N. Solopchenko \\ Saint-Petersburg State Polytechnical University \\ Vladik Kreinovich \\ The University of Texas at El Paso, vladik@utep.edu
}

Follow this and additional works at: https://scholarworks.utep.edu/cs_techrep

Part of the Computer Sciences Commons

Comments:

Technical Report: UTEP-CS-14-30a

Short version published in Proceedings of the International Conference on Advanced

Mathematical and Computational Tools in Metrology and Testing AMTCM'2014, St. Petersburg, Russia, September 9-12, 2014, Paper 088; full paper published in: Franco Pavese (ed.), Advanced Mathematical and Computational Tools in Metrology and Testing AMTCM'X, World Scientific, Singapore, 2015, pp. 340-348.

\section{Recommended Citation}

Semenov, Konstantin K.; Solopchenko, Gennady N.; and Kreinovich, Vladik, "Fuzzy Intervals As Foundation Of Metrological Support For Computations With Inaccurate Data" (2014). Departmental Technical Reports (CS). 832.

https://scholarworks.utep.edu/cs_techrep/832

This Article is brought to you for free and open access by the Computer Science at ScholarWorks@UTEP. It has been accepted for inclusion in Departmental Technical Reports (CS) by an authorized administrator of ScholarWorks@UTEP. For more information, please contact Iweber@utep.edu. 


\title{
FUZZY INTERVALS AS FOUNDATION OF METROLOGICAL SUPPORT FOR COMPUTATIONS WITH INACCURATE DATA
}

\author{
SEMENOV, K. K., SOLOPCHENKO, G. N. \\ Department of Measurement Informational Technologies, St. Petersburg State \\ Polytechnical University, 29, Polytechnicheskaya str., St. Petersburg, 195251, Russia \\ KREINOVICH, V. YA. \\ Department of Computer Science, University of Texas at El Paso, \\ $500 \mathrm{~W}$. University, El Paso, TX 79968, USA
}

\begin{abstract}
In this paper, we discuss the possibility of using the formalism of fuzzy intervals combined with automatic differentiation technique as a basis for numerical software selfverification in metrology. The natural domain of such approach is calculating indirect measurements results using the inaccurate results of direct measurements as the initial data. We propose to support software for such computations with tools that allow us to receive simultaneously calculated results and their error characteristics. Only such software can be put to metrological validation in full.
\end{abstract}

In many practical situations, the inaccurate results of direct measurements are used for calculations of indirect measurements results. Final data are also uncertain. Characteristics of this uncertainty should be expressed in quantitative form and presented together with indirect measurement result. The main purpose of this paper is to discuss ways to provide software for measured data processing with tools of automatic calculation of final result uncertainty. Only software that is supported in such manner can pass the metrological certification in full.

To achieve this purpose, we propose to use combination of two formalisms: fuzzy intervals approach - to represent inaccuracy of initial data for calculations, and formalism of software automatic differentiation - to compute how initial data uncertainty transforms to inherited uncertainty of final result.

There are many approaches for representing inaccuracy of measured data that act as initial information for subsequent calculations. Modern approaches take into account different information about the initial data inaccuracy. Some of them use random variables [1-3] for uncertainty representing and handling with it, other ones use bounds on possible values of initial data [4-6]. Interval representation of data inaccuracy was firstly mentioned by Wiener [7] and Kantorovich [8]. With the development of the fuzzy set theory, its formalism became actual tool for uncertainty expressing in metrology [9, 10]. Natural evolution of ideas of interval and fuzzy frameworks is the concept of fuzzy 
interval [9]. In this paper, we show that the combination of fuzzy interval approach with technique of automatic differentiation of programs is the most perspective way to achieve the declared purpose in metrology. This approach allows operating with both objective and subjective (expert) data that can occur in applications.

Let us consider advantages of using fuzzy intervals instead of the traditional intervals as a characteristic of uncertainty in computations with inaccurate data. Let $\tilde{x}_{1}=x_{1}+\Delta x_{1}, \ldots, \tilde{x}_{n}=x_{n}+\Delta x_{n}$ be the measurement results for quantities $x_{1}, \ldots, x_{n}$ that were obtained with absolute errors $\Delta x_{1}, \ldots, \Delta x_{n}$. Let $y=f\left(x_{1}, \ldots, x_{n}\right)$ be the function that describes the necessary computations. We should compute not only value $\tilde{y}=f\left(\tilde{x}_{1}, \ldots, \tilde{x}_{n}\right)=f\left(x_{1}+\Delta x_{1}, \ldots, x_{n}+\Delta x_{n}\right)$, but also characteristics of its inaccuracy:

$$
\Delta y=f\left(x_{1}+\Delta x_{1}, x_{2}+\Delta x_{2}, \ldots, x_{n}+\Delta x_{n}\right)-f\left(x_{1}, x_{2}, \ldots, x_{n}\right) .
$$

If the errors $\Delta x_{1}, \ldots, \Delta x_{n}$ are small, then we can simplify the problem by linearizing of the function $\tilde{y}=f\left(\tilde{x}_{1}, \ldots, \tilde{x}_{n}\right)$. In this case, the resulting inaccuracy becomes a linear combination of the errors $\Delta x_{1}, \ldots, \Delta x_{n}$ :

$$
\Delta y \approx \sum_{i=1}^{n} \frac{\partial f\left(\tilde{x}_{1}, \tilde{x}_{2}, \ldots, \tilde{x}_{n}\right)}{\partial x_{i}} \cdot \Delta x_{i}
$$

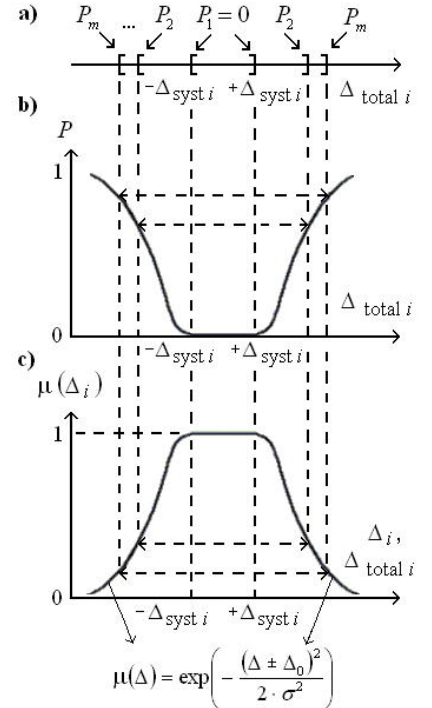

Fig. 1. Membership function construction for fuzzy interval
Since the computation of $f$ is performed by a computer program, we can estimate derivatives in Eq. 1 efficiently and with absolute accuracy using technique of automatic differentiation [11]. This technique is used in $[12,13]$ for solving a wide class of metrological problems.

Let us describe errors $\Delta x_{1}, \ldots, \Delta x_{n}$ as fuzzy variables. Operations of addition and multiplication with constant in Eq. 1 should be treated accordingly. Errors $\Delta x_{1}, \ldots, \Delta x_{n}$ are composed of systematic $\Delta_{\text {syst }} x_{1}, \ldots, \Delta_{\text {syst }} x_{n}$ and random $\Delta_{\text {rand }} x_{1}, \ldots, \Delta_{\text {rand }} x_{n}$ components. It should be considered that they act differently when we perform multiple measurements.

Usually, it is known from the technical documentation for measuring instruments that $\left|\Delta_{\text {syst }} x_{i}\right| \leq \Delta_{\text {syst } i}$ with probability $P_{\text {syst }}=1$ and 
that $\left|\Delta_{\text {rand }} x_{i}\right| \leq \Delta_{\text {rand } i}$ with probability greater than or equal to $P_{\text {rand }}<1$. So, inequality $\left|\Delta x_{i}\right|=\left|\Delta_{\text {syst }} x_{i}+\Delta_{\text {rand }} x_{i}\right| \leq \Delta_{\text {total } i}=\Delta_{\text {syst } i}+\Delta_{\text {rand } i}$ holds with probability $P>P_{\text {syst }} \cdot P_{\text {rand }}=P_{\text {rand }}$. The value $\Delta_{\text {total } i}$ of the total error bound is the function of confidence probability $P: \Delta_{\text {total } i}=\Delta_{\text {total } i}(P)$. If we associate the set of intervals $J_{1-P}=\left[\begin{array}{lll}-\Delta_{\text {total } i}(P), & \Delta_{\text {total } i}(P)\end{array}\right.$ with values $\alpha=1-P$ then the received curve $\alpha=\alpha\left(\Delta_{\text {total } i}\right)$ will correspond to membership function $\mu\left(\Delta_{i}\right)$ of a fuzzy interval that will represent information about total error (Fig. 1).

The curve $\mu\left(\Delta_{i}\right)$ is the symmetrical curvilinear trapezoid. Its upper base represents information about the systematic part of error and its lateral sides describe known information about the error's random component. The value $\alpha$ is the degree of belief of the statement "limit possible value of total error $\Delta x_{i}$ of measurement result $\widetilde{x}_{i}$ will be inside the interval $J_{\alpha}$ ".

In $[14,15]$, it is theoretically justified that the trapezoid $\alpha=\mu\left(\Delta_{i}\right)$ should has left and right halves of Gaussian curve as its latter sides (Fig. 1c). If experts produce membership function of another type then it can be easily approximated with function $\widetilde{\mu}\left(\Delta_{i}\right)$ of the necessary form. Really, let experts give two sets $\Delta_{i j}^{(1)} \leq-\Delta_{\text {syst } i}$ and $\Delta_{i j}^{(2)} \geq \Delta_{\text {syst } i}, j=1,2, \ldots, m$ of values that satisfy conditions $\mu\left(\Delta_{i j}^{(1)}\right)=\mu\left(\Delta_{i j}^{(2)}\right)=\alpha_{j}$, where $\alpha_{j}$ are pre-defined degrees of belief. Then parameters $\sigma^{(1)}$ and $\sigma^{(2)}$ of Gaussain curves for left and right sides of $\widetilde{\mu}\left(\Delta_{i}\right)$ can be estimated as $\max _{j} \frac{\left|\Delta_{i j}^{(1)}+\Delta_{\text {syst } i}\right|}{\sqrt{-2 \cdot \ln \alpha_{j}}}$ and $\max _{j} \frac{\left|\Delta_{i j}^{(2)}-\Delta_{\text {syst } i}\right|}{\sqrt{-2 \cdot \ln \alpha_{j}}}$ correspondingly. The final value $\sigma$ can be taken as $\max \left\{\sigma^{(1)}, \sigma^{(2)}\right\}$ for symmetrical membership function. Some examples of such approximations are presented on the Fig. 2.

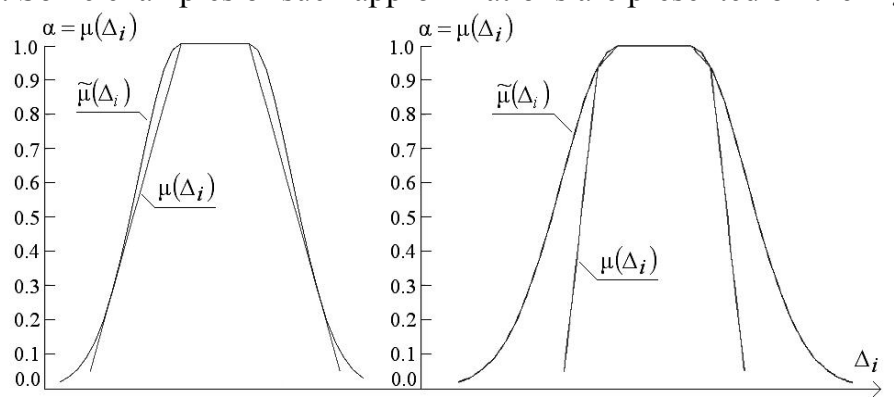

Fig. 2. Gaussian-type approximation for membership function of fuzzy interval constructed by expert evaluations 
So, membership function of fuzzy interval can be described with only two parameters $\left\{\Delta_{0}, \sigma\right\}$, where $\Delta_{0}$ is such a value that $\mu\left(\Delta_{i}\right)=1$ if $\left|\Delta_{i}\right| \leq \Delta_{0}$ and $\mu\left(\Delta_{i}\right)<1$ if $\left|\Delta_{i}\right|>\Delta_{0}$ and $\sigma$ is parameter of latter sides of $\mu\left(\Delta_{i}\right)$.

To process fuzzy intervals, different definitions of arithmetic operations can be used. Its rational choice depends on the concrete problem to solve. The general definition is the following. Let $\mu_{1}\left(\Delta_{1}\right)$ and $\mu_{2}\left(\Delta_{2}\right)$ be membership


result interval $\Delta_{\text {total } 3}=\Delta_{\text {total } 1}+\Delta_{\text {total } 2}$ will have membership function $\mu_{3}\left(\Delta_{3}\right)=\sup T\left(\mu_{1}\left(\Delta_{1}\right), \mu_{2}\left(\Delta_{2}\right)\right)$, where $T$ is triangular norm (see for $\Delta_{1}+\Delta_{2}=\Delta_{3}$

details [10]). For metrology, we should choose such norm $T$ or such algebraically closed family of suitable membership functions that can provide the decreasing of fuzziness when we average fuzzy intervals. If we choose widely used product triangular norm $T(a, b)=a \cdot b$ then it can be proved [15] that such family exists and the example of corresponding membership function is presented on Fig. 1. This class is closed for addition and multiplication with constant, as it is required in Eq.1. So, to process fuzzy intervals, we can process only tuples $\left\{\Delta_{0}, \sigma\right\}$ [16]. Linear operations with fuzzy intervals, which are used in Eq. 1, will lead to the following operations with tuples:

$$
\left\{\Delta_{01}, \sigma_{1}\right\} \pm\left\{\Delta_{02}, \sigma_{2}\right\}=\left\{\Delta_{01}+\Delta_{02}, \sqrt{\sigma_{1}^{2}+\sigma_{2}^{2}}\right\}, c \cdot\left\{\Delta_{01}, \sigma_{1}\right\}=\left\{c\left|\cdot \Delta_{01},\right| c \mid \cdot \sigma_{1}\right\} \text {. }
$$

We can see that these rules repeat well-known rules that are used in metrology for processing systematic errors and for standard deviations of random errors. From $[17,18]$, we can conclude that we should use values $\alpha=0.05 \div 0.10$ to get most credible confidence interval from fuzzy interval. As it was demonstrated in [16], averaging of fuzzy intervals for multiple measurements results reduces the uncertainty of its borders and makes it tend to the classical deterministic interval - in full correspondence with traditional metrology.

Alternative approach is to choose Lukasiewicz triangular norm $T(a, b)=\max \{a+b-1,0\}$ that is widely used too. In the manner of paper [15], we can prove that the only possible form of membership function should be symmetrical curvilinear trapezoid with parabolic lateral sides (left curve on Fig. 3).

Fig. 3 shows the following quantitative property of fuzzy interval. Let $\mu_{\text {syst }}\left(\Delta_{i}\right)$ and $\mu_{\text {rand }}\left(\Delta_{i}\right)$ be membership functions for fuzzy intervals that represent purely systematic $\Delta_{\text {syst }} x_{i}$ and purely random $\Delta_{\text {rand }} x_{i}$ components of total error $\Delta_{\text {total }} x_{i}=\Delta_{\text {syst }} x_{i}+\Delta_{\text {rand }} x_{i}$ of some quantity $\tilde{x}_{i}$. Then membership function of sum of these two fuzzy intervals is exactly $\mu\left(\Delta_{i}\right)$ that is membership function of fuzzy interval constructed for total error. Moreover, we always can 
break fuzzy interval for total error into sum of fuzzy intervals for systematic and random error components and such decomposition will be unique.



Fig. 3. Membership function of fuzzy interval corresponding to Lukasiewicz triangular norm

It can be easily shown [15] that analogous property takes place for the case of product triangular norm for membership functions with Gaussian lateral sides.

In the case of Lukasiewicz triangular norm membership function of fuzzy interval also can be described with only two parameters that is clear from Fig. 3. Linear operations with fuzzy intervals turn to rules for these parameters that repeat well-known rules in metrology as it was already stated for product norm. The main difference between Lukasiewicz and product triangular norms for considered problem is that fuzzy interval carrier is bounded or not.

If the examined triangular norms aren't applicable according to any circumstances then we can organize the new norm $T_{1}$ from product or Lukasiewicz norm $T_{0}$ using relationship $T_{1}\left(\mu_{1}, \mu_{2}\right)=\varphi^{-1}\left(T_{0}\left(\varphi\left(\mu_{1}\right), \varphi\left(\mu_{2}\right)\right)\right)$ where $\mu_{1}$ and $\mu_{2}$ are membership functions and operands, $\varphi$ is arbitrary increasing function that produces mapping $[0,1] \rightarrow[0,1], \varphi(0)=0, \varphi(1)=1$. Membership function family that can express fuzzy interval for norm $T_{1}$ can be organized by transformation $\varphi^{-1}$ applied to all elements of original function class for norm $T_{0}$.

Fuzzy interval description of measurement inaccuracy is in good agreement with known approaches [1-6] used for numerical software self-verification. It can be shown that the fuzzy intervals formalism is in good accordance with probabilistic [1] and interval [4] arithmetics.

Really, let fuzzy intervals represent the pure systematic error. Then all linear operations with them will be performed by interval arithmetic. It can be easily understood from operations with tuples for such a case:

$$
\left\{\Delta_{01}, 0\right\} \pm\left\{\Delta_{02}, 0\right\}=\left\{\Delta_{01}+\Delta_{02}, 0\right\} \text { and } c \cdot\left\{\Delta_{01}, 0\right\}=\left\{c \mid \cdot \Delta_{01}, 0\right\} \text {. }
$$

We see that these operations are identical to classic interval arithmetic. It was shown for product norm and can be shown for Lukasiewicz norm as well. 
Since interval arithmetic is used when we have the only limit values for quantities, we can state that proposed approach covers this important particular case.

If fuzzy data obtained from experts are impeachable or the expert quantity is insufficient then the only way to manage data uncertainty will be to obtain objective information by performing multiple measurements. In many practical applications probabilistic arithmetic [1] is used for this purpose. This is the tool to manage with imprecise distributions of random variables. It is based on objects called probabilistic boxes (p-boxes for short) that represent the domain of pos-sible values of cumulative distribution function $F(x)$. P-box can be expressed in the following form: $F(x) \in[F(x), \bar{F}(x)]$ for all possible values $x$ of random vari-able, where $\underline{F}(x)$ and $\bar{F}(x)$ are low and upper bounds of p-box. It is well known [1] that if we want to sum two variables (maybe correlated) represented by p-boxes $F_{1}(x) \in\left\lfloor F_{1}(x), \overline{F_{1}}(x)\right\rfloor$ and $F_{2}(x) \in\left[F_{2}(x), \overline{F_{2}}(x)\right\rfloor$ then the resulting $\mathrm{p}$-box will have the following bounds:

$$
F_{3}(z) \in\left[\sup _{z=x+y} \max \left\{\underline{F_{1}}(x)+\underline{F_{2}}(y)-1,0\right\} \quad \inf _{z=x+y} \min \left\{\overline{F_{1}}(x)+\overline{F_{2}}(y), 1\right\}\right] .
$$

Let $F^{-}(x) \in[F(x), \Phi(x)]$ be p-box to present the negative limit value of purely random component of measurement error. Here $\Phi(x)=\left\{\begin{array}{l}1, x>0 \\ 0, x \leq 0\end{array}\right.$ is Heaviside step function. Let $F^{+}(x) \in[\Phi(x), \bar{F}(x)]$ be p-box to present the positive limit value of the same random error. Then we can require symmetry in the following sense: $\bar{F}(x)=1-\underline{F}(-x)$. We suppose that there is no systematic error at all and that's why $\underline{F}(x)=1$ and $\bar{F}(x)=0$ if $x=0$. We can construct the membership function $\mu$ from these two p-boxes: $\mu(x)=\left\{\begin{array}{ll}\underline{F}(x), & x \leq 0 \\ 1-\bar{F}(x), & x>0\end{array}\right.$. Then the value $\alpha=\mu(x)$ of belief degree has the meaning that was stated above.

Let us choose the Lukasiewicz triangular norm. Let $\mu_{1}\left(\Delta_{1}\right)$ and $\mu_{2}\left(\Delta_{2}\right)$ be membership function of two fuzzy intervals constructed for purely random errors of quantities $\tilde{x}_{1}$ and $\tilde{x}_{2}$ (see the right curve on Fig. 3). Then the sum of these fuzzy intervals will have the membership function of the following type: $\mu_{3}\left(\Delta_{3}\right)=\sup _{\Delta_{1}+\Delta_{2}=\Delta_{3}} \max \left\{\mu_{1}\left(\Delta_{1}\right)+\mu_{2}\left(\Delta_{2}\right)-1,0\right\}$. Let $F_{1}^{-}\left(\Delta_{1}\right) \in\left[F_{1}(x), \Phi(x)\right]$, $F_{1}^{+}\left(\Delta_{1}\right) \in\left[\Phi(x), \overline{F_{1}}(x)\right]$ be the p-boxes corresponded to first fuzzy interval, $\left.F_{2}^{-}\left(\Delta_{2}\right) \in \underline{\left[F_{2}\right.}(x), \Phi(x)\right], F_{2}^{+}\left(\Delta_{2}\right) \in\left[\Phi(x), \overline{F_{2}}(x)\right]$ - to second fuzzy interval and 
$F_{3}^{-}\left(\Delta_{3}\right) \in\left[F_{3}(x), \Phi(x)\right], F_{3}^{+}\left(\Delta_{3}\right) \in\left[\Phi(x), \overline{F_{3}}(x)\right]-$ to resulting fuzzy interval. For values $\Delta_{3} \leq 0$ we see the total identity between fuzzy approach and probabilistic arithmetic: $\mu_{3}\left(\Delta_{3}\right)=\sup _{\Delta_{1}+\Delta_{2}=\Delta_{3}} \max \left\{\mu_{1}\left(\Delta_{1}\right)+\mu_{2}\left(\Delta_{2}\right)-1,0\right\}=$ $=\sup _{\Delta_{1}+\Delta_{2}=\Delta_{3}} \max \left\{\underline{F_{1}}\left(\Delta_{1}\right)+\underline{F_{2}}\left(\Delta_{2}\right)-1,0\right\}$.

Let us examine values $\Delta_{3}>0$ :

$$
\begin{gathered}
\mu_{3}\left(\Delta_{3}\right)=\sup _{\Delta_{1}+\Delta_{2}=\Delta_{3}} \max \left\{\mu_{1}\left(\Delta_{1}\right)+\mu_{2}\left(\Delta_{2}\right)-1,0\right\}, \\
1-\overline{F_{3}}\left(\Delta_{3}\right)=\sup _{\Delta_{1}+\Delta_{2}=\Delta_{3}} \max \left\{1-\overline{F_{1}}\left(\Delta_{1}\right)-\overline{F_{2}}\left(\Delta_{2}\right), 0\right\}, \\
\overline{F_{3}}\left(\Delta_{3}\right)=1-\sup _{\Delta_{1}+\Delta_{2}=\Delta_{3}}\left(1-\min \left\{\overline{F_{1}}\left(\Delta_{1}\right)+\overline{F_{2}}\left(\Delta_{2}\right), 1\right\}\right)= \\
=\inf _{\Delta_{1}+\Delta_{2}=\Delta_{3}}\left(\min \left\{\overline{F_{1}}\left(\Delta_{1}\right)+\overline{F_{2}}\left(\Delta_{2}\right), 1\right\}\right) .
\end{gathered}
$$

We established the connection between fuzzy intervals and p-boxes formalisms for metrological applications. To postulate relationship with other approaches for dealing with uncertainty, let us cite paper [19] that notes equivalence of probabilistic arithmetic with series of other formalisms.

Let us examine the example of constructing fuzzy interval from empirical data. Let $\tilde{x}_{i j}$ be values of multiple measurements results of one quantity, $j=1,2, \ldots m$. We should obtain fuzzy interval for uncertainty of its value. From technical documentation on used measuring instrument we can find out the bound $\Delta_{\text {syst } i}$ for possible systematic error of every $\tilde{x}_{i j}$. Then we can estimate bounds $\Delta_{\text {rand } i}$ of possible random error of obtained mean value $\widetilde{x}_{i}=\frac{1}{m} \cdot \sum_{j=1}^{m} \widetilde{x}_{i j}: \Delta_{\text {rand } i}=\frac{s}{\sqrt{m}} \cdot t_{0,975}(m-1)$, where $t_{0,975}(m-1)$ is $97,5 \%$ quantile of Student's distribution with parameter equal to $(m-1)$, $s^{2}=\frac{1}{m-1} \cdot \sum_{j=1}^{m}\left(\tilde{x}_{i j}-\tilde{x}_{i}\right)^{2}$ is dispersion estimate for random error population. The probability compared with this confidence interval is $P=95 \%$.

Let us choose product triangular norm, then we can easily construct fuzzy interval for $\tilde{x}_{i}$ from its tuple: $\left\{\Delta_{0}, \sigma\right\}=\left\{\Delta_{\text {syst } i}, \frac{s \cdot t_{0.5 \cdot(1+P)}(m-1)}{\sqrt{-2 \cdot m \cdot \ln (1-P)}}\right\}$. The value of Gaussian parameter $\sigma$ is determined from the following consideration: 
nested interval $J_{1-P}$ for fuzzy interval on the degree of belief equal to $(1-P)$ must be of form $\left[-\Delta_{\text {syst } i}-\Delta_{\text {rand } i}, \Delta_{\text {syst } i}+\Delta_{\text {rand } i}\right\rfloor$.

We can see again that all operations performed to construct fuzzy interval don't go beyond the scope of traditional metrology and its approaches.

From the results of paper [15], it can be concluded that the natural domain for using fuzzy intervals is limited to linear operations. Attempts of their using for nonlinear transforms lead to difficulties. That's why it is reasonable to use fuzzy intervals jointly with automatic differentiation techniques. Program code example for simple realization of automatic differentiation for real-valued function is presented in [20] correspondingly to metrological problems.

Let us turn back to Eq. 1. The partial derivatives $\partial f / \partial x_{i}$ of the function $f$ are computed accurately but at inaccurate values $\widetilde{x}_{1}, \ldots, \widetilde{x}_{n}$. Thus, the value of $\Delta y$ can be underestimated because we use linearization for $f$ in a slightly different domain: we take the domain $\left[\widetilde{x}_{1}-\Delta_{1}, \widetilde{x}_{1}+\Delta_{1}\right] \times \ldots \times\left[\widetilde{x}_{n}-\Delta_{n}, \widetilde{x}_{n}+\Delta_{n}\right]$ instead of $\left[x_{1}-\Delta_{1}, x_{1}+\Delta_{1}\right] \times \ldots \times\left[x_{n}-\Delta_{n}, x_{n}+\Delta_{n}\right]$. To prevent this situation, we can use the following approach. If the automatic differentiation is used to estimate first-order derivatives, then we can apply this technique again (recursively) to obtain the values of the second-order derivatives. If the errors $\Delta x_{1}, \ldots, \Delta x_{n}$ are small enough, then the following inequality will hold:

$$
\left|\frac{\partial f\left(x_{1}, x_{2}, \ldots, x_{n}\right)}{\partial x_{i}}-\frac{\partial f\left(\widetilde{x}_{1}, \widetilde{x}_{2}, \ldots, \widetilde{x}_{n}\right)}{\partial x_{i}}\right| \leq \sum_{j=1}^{n}\left|\frac{\partial^{2} f\left(\widetilde{x}_{1}, \widetilde{x}_{2}, \ldots, \widetilde{x}_{n}\right)}{\partial x_{i} \partial x_{j}}\right| \cdot \Delta_{\text {total } j} .
$$

This improves Eq. 1 and allows us to obtain more correct results. For that we should use in Eq. 1 upper bounds on derivatives absolute values that can be produced with the inequality above:

$$
|\Delta y| \leq \sum_{i=1}^{n}\left[\left|\frac{\partial f\left(\tilde{x}_{1}, \widetilde{x}_{2}, \ldots, \tilde{x}_{n}\right)}{\partial x_{i}}\right|+\sum_{j=1}^{n}\left|\frac{\partial^{2} f\left(\widetilde{x}_{1}, \widetilde{x}_{2}, \ldots, \widetilde{x}_{n}\right)}{\partial x_{i} \partial x_{j}}\right| \cdot \Delta_{\text {total } j} \cdot\right] \cdot\left|\Delta x_{i}\right| .
$$

In this work, we show that it is possible to realize such software that can present simultaneously final result and its uncertainty characteristics. For this, it is proposed to use combination of fuzzy interval formalism and automatic differentiation technique. Realization of this approach requires very small modifications in software initial code [21].

\section{References}

1. R. Williamson and T. Downs. International Journal of Approximate Reasoning. 4, 89 (1990).

2. D. Berleant and C. Goodman-Strauss. Reliable Computing. 4 (2), 147 (1998). 
3. S. Ferson, V. Kreinovich, L. Ginzburg, D. Myers and K. Sentz. Constructing probability boxes and Dempster-Shafer structures, Technical report SAND2002-4015 (2003).

4. R. Moore. Methods and Applications of Interval Analysis . (1979).

5. L. de Figueiredo and J. Stolfi. Numerical Algorithms. 37 (1-4), 147 (2004).

6. M. Berz and G. Hoffstätter. Reliable Computing. 4 (1), 83 (1998).

7. N. Wiener. Proc. of the London Mathematical Society, 19, 185 (1921).

8. L. Kantorovich. Siberian Mathematical Journal. 3, 701 (1962). In Russian.

9. L. Reznik, W. Jonson and G. Solopchenko. Proc. NASA Conf. NAFIPS'94. 405 (1994).

10. S. Salicone. Measurement Uncertainty. An approach via the Mathematical Theory of Evidence. Springer Series in Reliability Engineering (2007).

11. G. Corliss, C. Faure, A. Griewank, L. Hascoët and U. Naumann. Automatic Differentiation of Algorithms: From Simulation to Optimization.383 (2002).

12. B. Hall. Measurement Science and Technology. 13 (4), 421 (2002).

13. L. Mari. Measurement. 42 (6), 844 (2009).

14. V. Kreinovich, C. Quintana and L. Reznik. Gaussian membership functions are most adequate in representing uncertainty in measurements. Technical Report, University of Texas at El-Paso (1992).

15. K. Semenov. Informatics and its applications. 6 (2), 101 (2012). In Russian. 16. V. Kreinovich, L. Reznik, K. Semenov and G. Solopchenko. Proceedings of XX IMEKO World Congress. paper IMEKO-WC-2012-ADC-O3 (2012).

17. H. Nguen, V. Kreinovich, C.-W. Tao, G. Solopchenko. Soft Computing in Measurement and Information Acquisition. 10 (2003).

18. P. Novitskiy and I. Zograf. Errors estimation for measurements results. (1991). In Russian.

19. H. Regan, S. Ferson, D. Berleant. Equivalence of methods for uncertainty propagation of real-value random variables. International Journal of Approximate Reasoning. 36 (1) (2004).

20. K. Semenov. Metrological aspects of stopping iterative procedures in inverse problems for static-mode measurements. This book.

21. K. Semenov, G. Solopchenko. Theoretical prerequisites for implementation of metrological self-tracking of measurement data analysis programs. Measurement techniques. 53 (6), 592 (2010). 